09.02 - Physiotherapists

\title{
23755
}

\section{Functional ability in patients with COPD}

COPD, Extrapulmonary impact, Chronic diseases
A. F. Machado ${ }^{1}$
S. Souto-Miranda ${ }^{1}, A$
A. Tavares ${ }^{1}, A$
A. Oliveira ${ }^{1}$, C. Jácome ${ }^{2}$, J. Cruz $^{1}$, B. Cabrita $^{3}$, C. Cascais Costa ${ }^{4}$, C. Valente ${ }^{4}$, V. Martins ${ }^{5}$, L. Andrade ${ }^{4}$, P. Simão ${ }^{3}$, A. Marques ${ }^{1}$
${ }^{1}$ Lab3R - Respiratory Research and Rehabilitation Laboratory, School of Health Sciences, University of Aveiro (ESSUA) - Aveiro (Portugal), ${ }^{2}$ CINTESIS -Center for Health Technology and Services Research, Faculty of Medicine, University of Porto - Porto (Portugal), ${ }^{3}$ Pulmonology Department, Unidade Local de Saúde de Matosinhos - Matosinhos (Portugal), ${ }^{4}$ Pulmonology Department, Centro Hospitalar do Baixo Vouga - Aveiro (Portugal), ${ }^{5}$ Pulmonology Department, Hospital Distrital Figueira Foz, Figueira da Foz - Figueira da Foz (Portugal)

Background: Functional impairment is a critical consequence of COPD. The 1-minute sit-to-stand (1STS) is a valid and reliable test to measure this health domain in patients with COPD, with established reference values. We aimed to quantify the prevalence of functional impairment in patients with COPD and identify the distribution of this impairment across disease stages.

Methods: A cross-sectional study with patients with COPD and healthy age-/gender-matched controls was conducted. Spirometry, the modified British Medical Research Council questionnaire (mMRC) and the 1STS were collected. Patients were grouped by the Global Initiative for Chronic Obstructive Lung Disease (GOLD) classifications. Differences between groups were explored with Kruskal-Wallis and Bonferroniadjusted pairwise comparisons.

Results: 366 patients with COPD (76\% male, $67 \pm 10 y$, FEV1 56 $\pm 21 \mathrm{pp})$ and 345 controls $(70 \%$ male, $67 \pm 9 y$, FEV $104 \pm 21 \mathrm{pp}$ ) participated. $50 \%$ of the patients presented functional impairment (i.e., performed below $80 \%$ predicted). Patients with COPD presented lower functional ability than controls, independently of the GOLD grade/group (Fig. 1). The most symptomatic patients (i.e., GOLD B and D) had the lowest functional levels.

Conclusion: Functional ability is reduced in patients with COPD in all GOLD grades/groups, especially in 
the most symptomatic. Future studies should explore therapeutic interventions to address this problem.
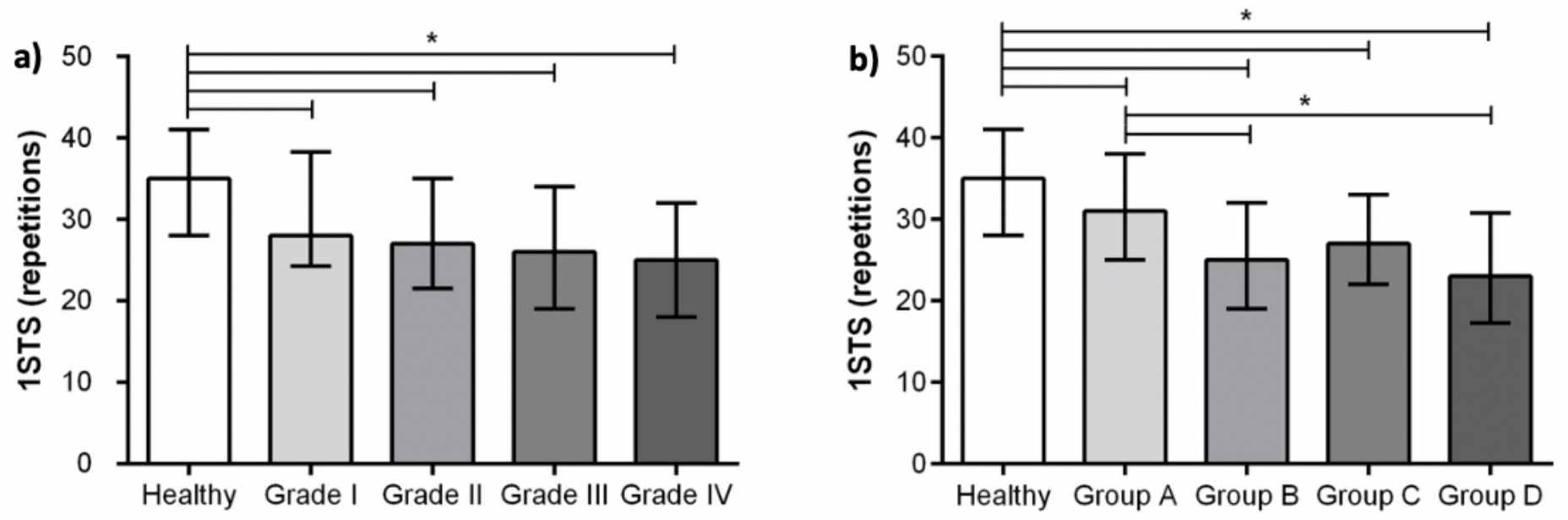

Figure 1 - Median number of repetitions in the 1-minute sit-to-stand (1STS) with interquartile range in: a) healthy controls vs. patients with COPD across Global Initiative for Chronic Obstructive Lung Disease (GOLD) spirometric grades (i.e., I, II, III, IV); b) healthy controls vs. patients with COPD across GOLD groups (i.e., A, B, C, D); ${ }^{*} \mathrm{p}<0.05$. 